\title{
Single-Case-Design Study of Finger-to-Axilla Compression Bandaging for Edema of the Hemiplegic Upper Limb
}

\author{
Louise Gustafsson, Jessica Lunnon, Melanie Hoyle, Kathryn Marshall, \\ Kylie Bower
}

KEY WORDS

- compression bandages

- edema

- hemiplegia

- upper extremity

- treatment outcome
Louise Gustafsson, PhD, is Associate Professor, School of Health and Rehabilitation Sciences, The University of Queensland, St. Lucia, Brisbane, Queensland, Australia; I.gustafsson@uq.edu.au

Jessica Lunnon was Occupational Therapy Student, School of Health and Rehabilitation Sciences, The University of Queensland, St. Lucia, Brisbane, Queensland, Australia.

Melanie Hoyle, MOccThySt, is Associate Lecturer, School of Health and Rehabilitation Sciences, The University of Queensland, St. Lucia, Brisbane, Queensland, Australia.

Kathryn Marshall is Occupational Therapist, Department of Occupational Therapy, Princess Alexandra Hospital, Woolloongabba, Queensland, Australia.

Kylie Bower is Occupational Therapist, Department of Occupational Therapy, Princess Alexandra Hospital, Woolloongabba, Queensland, Australia.
OBJECTIVE. This single-case study investigated the effectiveness of compression bandaging from the finger to the axilla in reducing poststroke edema in the upper limb.

METHOD. Repeated circumferential measurements were recorded at five points along the participants' hand and forearm. Analysis of the data included the generation of graphs, celeration lines, and visual analysis. RESULTS. Five participants with edema (mean 38 days poststroke) were recruited to the study. Fluctuations in edema were observed in all three study phases, with an increasing to decreasing trend in edema between the baseline and intervention phases. There was no clear trend from the intervention to second baseline phase.

CONCLUSION. Bandaging from the fingers to the axilla appears to be effective in reducing edema in the hand and forearm. However, return of edema after removal of the bandaging suggest that a greater understanding of underlying mechanisms and the appropriate intervention protocols is warranted.

Gustafsson, L., Lunnon, J., Hoyle, M., Marshall, K., \& Bower, K. (2016). Single-case-design study of finger-to-axilla compression bandaging for edema of the hemiplegic upper limb. American Journal of Occupational Therapy, 70, 7002210010. http://dx.doi.org/10.5014/ajot.2016.019125

Deople presenting with hemiplegia after stroke often develop edema in the hand, arm, or both as a result of weakness and immobility in the paretic limb (Gebruers, Truijen, Engelborghs, \& De Deyn, 2011; National Stroke Foundation, 2010). Edema of the upper limb negatively affects people's functioning (Boomkamp-Koppen, Visser-Meily, Post, \& Prevo, 2005) and may be associated with symptoms such as heaviness, stiffness, and reduced active motion of the hand and arm (Geurts, Visschers, van Limbeek, \& Ribbers, 2000; Post, Visser-Meily, Boomkamp-Koppen, \& Prevo, 2003). These symptoms slow down the rehabilitation process and also affect the person's ability to manage daily life activities (Gracies et al., 2000). Poststroke edema is a form of dependency edema caused by immobility and the absence of a muscle pump (Wigg \& Jones, 2006). Although poststroke edema is not the same as lymphedema (Geurts et al., 2000), some commonalities between their causes have been proposed (Benbow, 2009; Wigg \& Jones, 2006).

All edema develops as a result of a physiological imbalance between capillary filtration and lymph drainage (Badger, Peacock, \& Mortimer, 2000). In both poststroke edema and lymphedema, these dysfunctions lead to an imbalance of fluid exchange within the body, resulting in the accumulation of excess fluid and changes in the size and shape of various tissues in the limbs (Benbow, 2009). The difference between poststroke edema and lymphedema lies in the state of the underlying lymphatics. Whereas lymphedema is a chronic condition, 
poststroke edema is a temporary deficit in the lymphatic system's ability to remove excess fluid, and it generally subsides once movement is regained (Benbow, 2009).

\section{Interventions for Edema}

Suggested treatments to reduce poststroke edema of the extremities include continuous passive motion with elevation, electrical stimulation, and use of dynamic pressure garments (Foley, Mehta, Jutai, Staines, \& Teasell, 2013; National Stroke Foundation, 2010). A survey of 55 occupational therapists with varying levels of experience found that $58 \%$ were using compression bandaging as a treatment to prevent or reduce poststroke edema at the wrist, and 53\% were using it for the same purpose at the elbow (Gustafsson \& Yates, 2009). Despite the broad use of this technique by occupational therapists, the research evidence and recommendations regarding the use of compression bandaging as an intervention to reduce poststroke edema in the extremities are limited.

Although the established evidence for the effective treatment of poststroke edema is minimal, various effective strategies for the management of lymphedema have been clearly established in research and clinical practice. They include compression bandaging, compression garments, exercise, and manual or simple lymphatic drainage (massage; Benbow, 2009; Wigg \& Jones, 2006). Compression bandaging is specifically used to reduce swelling because bandages provide a rigid casing that the muscles can work against to improve the shape and size of the swelling (Benbow, 2009). This external pressure on the affected limb works to increase the efficiency of the muscle pump and decrease the production and buildup of fluid (Cohen, Payne, \& Tunkel, 2001. Using bandages to wrap the affected limb encourages lymphatic flow of the excess fluid out of the limb by activating and working with the muscle pump (Cohen et al., 2001).

\section{Current Study}

This study is the second part of a three-part research program investigating the effectiveness of various methods of compression for the management of poststroke edema in the upper limb. The first study investigated the difference between the use of low-stretch and high-stretch bandaging of the hand and found that both types of bandage were effective in reducing poststroke edema (Gustafsson, Walter, Bower, Slaughter, \& Hoyle, 2014). However, increases occurred in edema proximal to the hand during the intervention phase, as the edema shifted into the forearm. This edema subsequently returned to the hand after the bandages were removed. It was hypothesized that the proximal shift in edema, and the subsequent return of edema to the hand, may be minimized by bandaging the arm as well as the hand. Therefore, the aim of this study was to investigate the effectiveness of compression bandaging from the fingers to the axilla in reducing poststroke edema in the upper limb. The research question asked whether bandaging of the entire upper limb reduced edema measures in the hand and forearm.

\section{Method}

An ABA research design was used with a series of single casestudy participants. Ethical approval was obtained from the hospital and university ethics committees.

\section{Participants}

Participants for this study were recruited from an inpatient ward of a major metropolitan hospital in Brisbane, Queensland, Australia. Potential participants were eligible for inclusion in this study if they had upper-limb edema, defined as visually observable swelling in comparison with the unaffected upper limb, after a stroke; had the cognitive and communicative ability to consent to participation in the study; and were medically stable. Participants were excluded if they had any vascular or medical condition that contraindicated the use of compression bandaging.

\section{Instrumentation}

Edema measurement tape was used to measure the circumference of the middle phalanx of the third finger, the metacarpophalangeal joints, the wrist at the ulnar styloid, the ulnar styloid plus $10 \mathrm{~cm}$, and the ulnar styloid plus $20 \mathrm{~cm}$. The validity of the use of measurement tape has been established by Karges, Mark, Stikeleather, and Worrell (2003), who found that calculating volume using measurement tape had a high concurrent validity (intraclass correlation coefficient $=.99$ ) compared with the upper-limb water displacement method, which is the gold standard used in lymphedema measurement.

The reliability of measurement tape for this purpose has also been established, with evidence indicating that interrater reliability is good but can be maximized if all raters are trained to use the instrument in the same way (Lymphoedema Framework, 2006; te Slaa et al., 2011). All measurers were trained to use the same method when measuring the circumference of the participants' upper limb to improve the interrater reliability of this method. Each participant was assigned a consistent measurer, and measurements were all taken at the same time of day to ensure continuity of results. 


\section{Intervention}

A short stretch cohesive bandage was applied to each participant's hand, and a low-stretch compression bandage was applied up the arm to the axilla. Given the limited evidence for the use of bandages to reduce poststroke edema, the bandaging technique used by the therapist was drawn from established lymphedema evidence. Each therapist was trained to use multilayer lymphedema bandaging to bandage the participants' limb. This technique is recommended for the reduction of edema in the upper limb in the Lymphoedema Foundation's (2006) Best Practice for the Management of Lymphoedema guidelines.

\section{Procedures}

Potential participants were identified by the treating occupational therapists and provided with the participant information sheet. A member of the research team was available to discuss the study further if required. Informed consent was obtained from all participants. Demographic details were recorded at the time of recruitment with respect to the number of days since stroke, arm affected, dominant arm, hemisphere of stroke, type of edema, presence of active movement in the affected upper limb, presence of hypertonicity, and use of splints.

In the first baseline phase (Days 1-3), no compression was applied, and daily measurements were recorded for 3 consecutive days. A 3-day baseline was chosen due to ethical concerns about withholding treatment and as a minimally required timeframe to establish the pattern of the edema. In the intervention phase (Days 3-10), compression bandages were applied after the final baseline measurements were taken on Day 3. The bandages were removed to allow measurement of edema before reapplication on three occasions between Days 3 and 10. The measurement on Day 10 was the final measurement point for the intervention phase, and the bandages were not reapplied. After Day 10, in the second baseline phase, compression was removed, and follow-up measurements were recorded on Days 13, 15 , and 17.

\section{Data Analysis}

Each of the participants' measurements across the three phases were graphed to allow preliminary visual analysis of trend, slope, and level. Celeration lines were calculated manually following the procedure outlined by Portney and Watkins (2009) and drawn on the graphical representation of the results to assist with visual analysis. Celeration lines were positive, indicating an increasing trend, or negative, indicating a decreasing trend, and show the trend of data behavior within a specified time period.

\section{Results}

Six participants were eligible and agreed to participate in the study, but 1 participant did not complete the study protocol. The results for the 5 participants who completed the study protocol are reported. The 5 participants were recruited a mean of 38 days after stroke (standard deviation $=14$ ) and showed no evidence of active movement return. Two participants' left upper limb was affected, and 3 participants' right upper limb was affected. Three participants demonstrated some hypertonicity in the affected upper limb, and 2 participants wore a hand splint at night. The calculated celeration lines for participants are reported in Table 1.

\section{Within-Phase Trends}

All participants demonstrated fluctuating levels of edema in the baseline phase. They showed a predominantly increasing trend in edema during the first baseline phase with the exception of Participant 1 (see Figure 1). During the intervention phase, the level of edema fluctuated, with a general decreasing trend at all measurement points with the exception of Participant 1 at the phalanx and wrist and Participant 4 at the wrist. The fluctuations in the edema continued to be evident during the second baseline phase, with the results showing an increasing, decreasing, or static trend.

Table 1. Celeration Lines

\begin{tabular}{|c|c|c|c|c|c|}
\hline \multirow[b]{2}{*}{ Location } & \multicolumn{5}{|c|}{ Participant } \\
\hline & 1 & 2 & 3 & 4 & 5 \\
\hline \multicolumn{6}{|l|}{ Phalanx } \\
\hline Baseline & -1.012 & -1.007 & 1.012 & -1.006 & 1.026 \\
\hline Intervention & 1.018 & 1.000 & -1.019 & -1.031 & -1.064 \\
\hline Baseline & 1.000 & 1.016 & 1.006 & 1.019 & 1.028 \\
\hline \multicolumn{6}{|l|}{ MCP } \\
\hline Baseline & -1.024 & 1.014 & 1.002 & 1.006 & -1.002 \\
\hline Intervention & -1.020 & -1.034 & -1.008 & -1.007 & -1.021 \\
\hline Baseline & -1.040 & 1.005 & 1.013 & 1.020 & 1.000 \\
\hline \multicolumn{6}{|c|}{ Wrist at ulnar styloid } \\
\hline Baseline & 1.000 & -1.029 & 1.005 & 1.003 & 1.008 \\
\hline Intervention & -1.005 & -1.029 & -1.018 & 1.034 & -1.020 \\
\hline Baseline & -1.005 & 1.000 & 1.028 & -1.031 & 1.009 \\
\hline \multicolumn{6}{|c|}{ Ulnar styloid $+10 \mathrm{~cm}$} \\
\hline Baseline & -1.019 & -1.002 & 1.017 & 1.011 & 1.012 \\
\hline Intervention & -1.015 & -1.030 & -1.008 & -1.028 & -1.047 \\
\hline Baseline & -1.002 & -1.002 & 1.031 & -1.036 & 1.012 \\
\hline \multicolumn{6}{|c|}{ Ulnar styloid $+20 \mathrm{~cm}$} \\
\hline Baseline & -1.014 & 1.012 & 1.015 & 1.009 & -1.019 \\
\hline Intervention & 1.021 & -1.030 & -1.013 & -1.050 & -1.019 \\
\hline Baseline & -1.017 & 1.003 & 1.028 & -1.009 & 1.000 \\
\hline
\end{tabular}

Note. A positive celeration line indicates increasing edema; a negative celeration line indicates decreasing edema. MCP = metacarpophalangeal. 


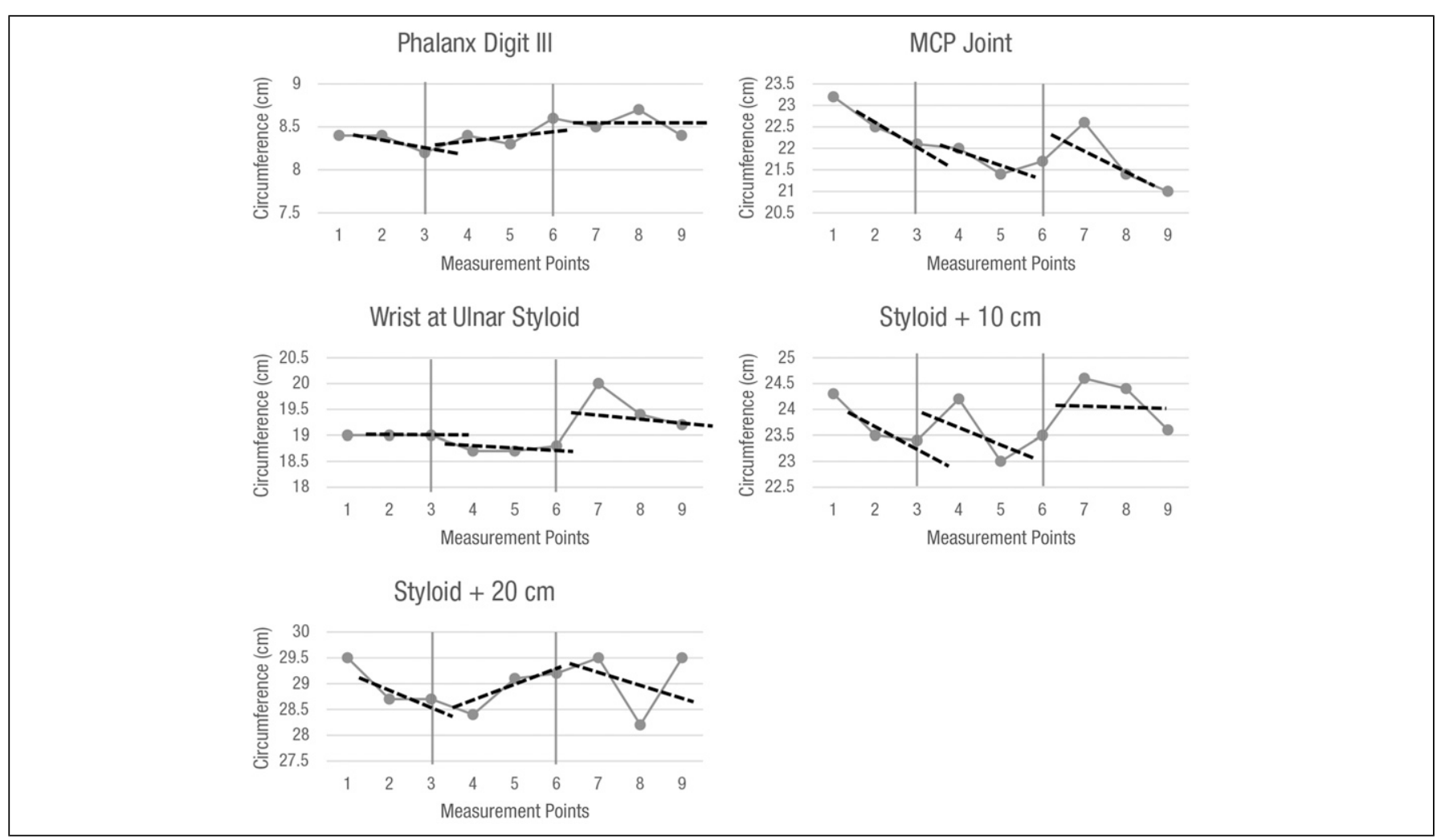

Figure 1. Participant 1.

Note. MCP = metacarpophalangeal.

\section{Between-Phase Trends}

Participants 2, 3, and 5 demonstrated a clear intervention effect from the application of the bandages, with an increasing trend during the first baseline phase changing to a decreasing trend at the majority of measurement points during the intervention phase, followed by an increasing trend during the second baseline phase (Figure 2).

Participant 1 performed differently, with a decreasing trend during the first baseline phase that continued into the intervention phase, except in the phalanx and styloidplus-20-cm measurement point. A predominantly decreasing trend was then found with the removal of the bandages (see Figure 1). Participant 4 had a predominantly increasing trend at baseline that reversed into a decreasing trend with bandages. The removal of the bandages resulted in an increasing trend at distal measures, but the proximal measures continued to decrease (Figure 3).

\section{Discussion}

The aim of this study was to investigate the effectiveness of compression bandaging from the fingers to the axilla in reducing poststroke edema in the upper limb. The fluctuating nature of edema reported in Gustafsson et al. (2014) was again evident in the participants recruited for this study. The results supported the effectiveness of bandaging from the fingers to the axilla in reducing edema at all measurement points during the intervention phase, in contrast to the previous findings that compression bandaging of the hand resulted in increases in edema in the forearm (Gustafsson et al., 2014).

Before discussing the results further, it is important to acknowledge the limitations of this study. It was a pragmatic, single-case-design study run in one treatment center. It was designed as a pilot trial to explore the application of compression bandaging for poststroke edema and included a relatively small number of participants, which limits our capacity to make conclusive statements regarding the intervention. However, it is one of the few studies to explore the effectiveness of compression bandaging for poststroke edema and should be used to guide further research.

The effectiveness of compression bandaging in reducing edema has previously been established and recommended for the management of lymphedema. This study's results suggest that the method may also be effective in reducing poststroke edema and may extend the previous findings (Gustafsson et al., 2014), with a trend for better results when bandaging from the fingers to the axilla, which suggests that consideration should be given to the extent of bandaging. Cohen et al. (2001) found that effective compression bandaging works to encourage the limb's lymphatic flow and, as a result, excess fluid or edema flows out of the limb. When bandaging the hand 


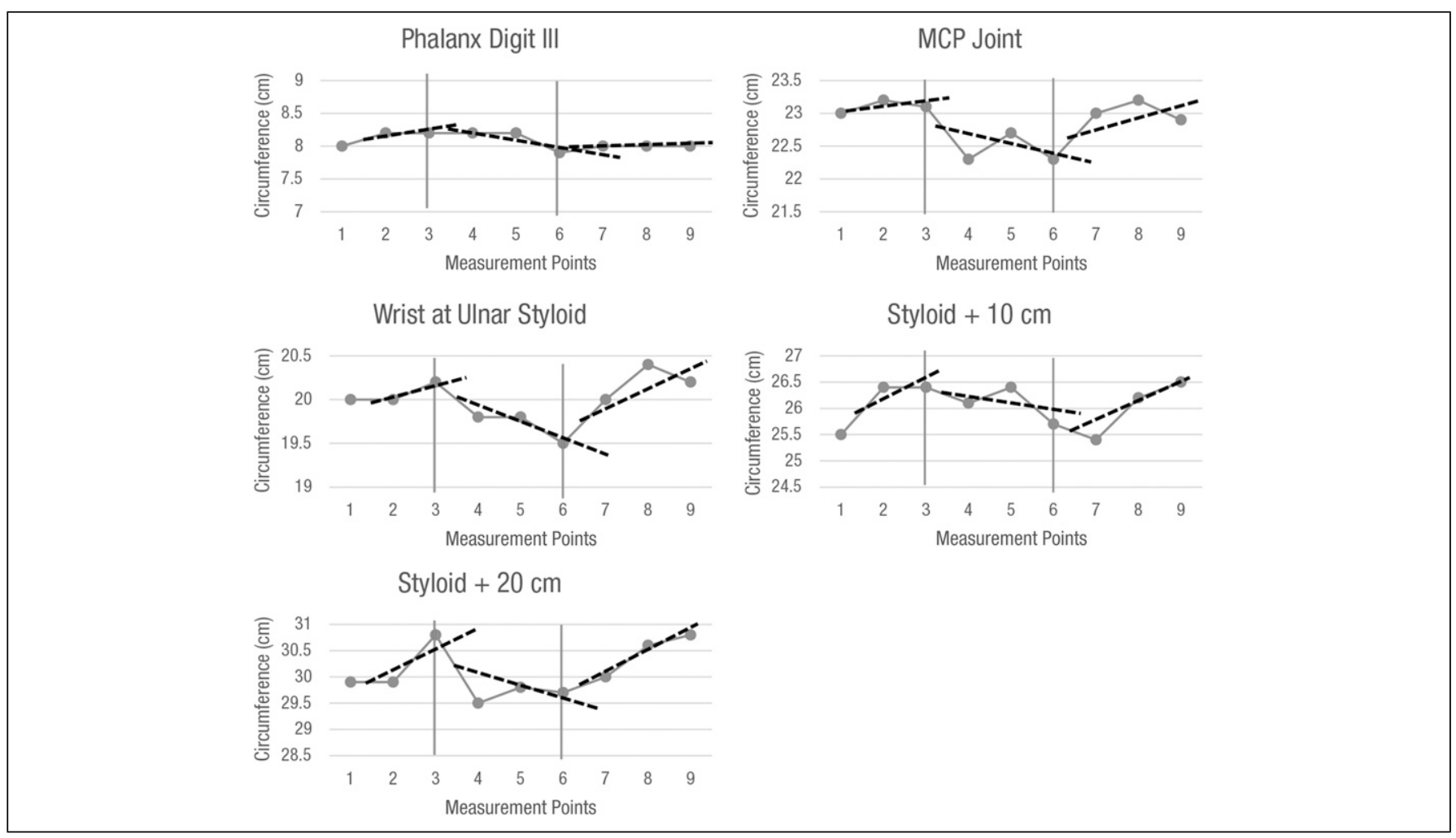

Figure 2. Participant 3.

Note. MCP = metacarpophalangeal.

alone, lymphatic flow appeared to be activated only in the hand, resulting in edema shifting out of the hand only to pool in the area immediately proximal to where the bandaging ended. When the upper limb was bandaged from the fingers to the axilla, lymphatic flow appeared to be activated throughout the entire limb, encouraging excess fluid removal from the hand and the forearm. However, this pattern was not evident for all participants, with some variations noted. It is important that the mechanism of edema after stroke be better understood to determine whether contributing factors differ among participants. Understanding these factors will allow clinicians to make informed decisions about the most appropriate compression technique or intervention for specific clients.

The return of the edema after the removal of the bandages suggests that further research is required to understand the ideal protocol for bandaging or the use of compression. For the purposes of this trial, a standard protocol was implemented that may not have included an intervention phase sufficiently long enough for the individualized needs of all participants. The continuing decreasing trend in edema for Participants 1 and 4 suggests that the timeline may have been sufficiently long to facilitate natural mechanisms. A further consideration for the remaining participants is the application of compression garments to maintain the reductions in edema. The lymphedema literature has indicated that edema return after a series of bandaging is well recognized, and prevention should include the application of a compression garment to the affected limb after cessation of bandaging procedures (Benbow, 2009; Cheville et al., 2003; Cohen et al., 2001). Investigating the effectiveness of compression bandaging followed by compression garments in reducing poststroke edema, compared with compression bandaging alone, was an area of further study and was the third and final stage of this program of research (Gustafsson, Patterson, Marshall, Bennett, \& Bower, 2016).

A research group from the Netherlands recently published a randomized controlled trial investigating a protocol for poststroke edema that included splinting, cryotherapy, high-stretch bandaging, and compression garments (Kuppens, Pijlman, Hitters, \& van Heugten, 2014). The results supported the fluctuating nature of edema reported here but did not achieve a result in favor of the protocol, possibly because of baseline differences between the two treatment groups. Regardless, the Kuppens et al. (2014) study highlighted that in the absence of evidence, clinicians are choosing to apply best practices to develop protocols for the management of edema. Practice context can influence the practices that are adopted, and clinical practice in both the Netherlands study and the hospital involved in this study appears to have evolved to a protocol in which a bandage was applied to the hand alone. The application of compression bandages to the entire upper limb may be 


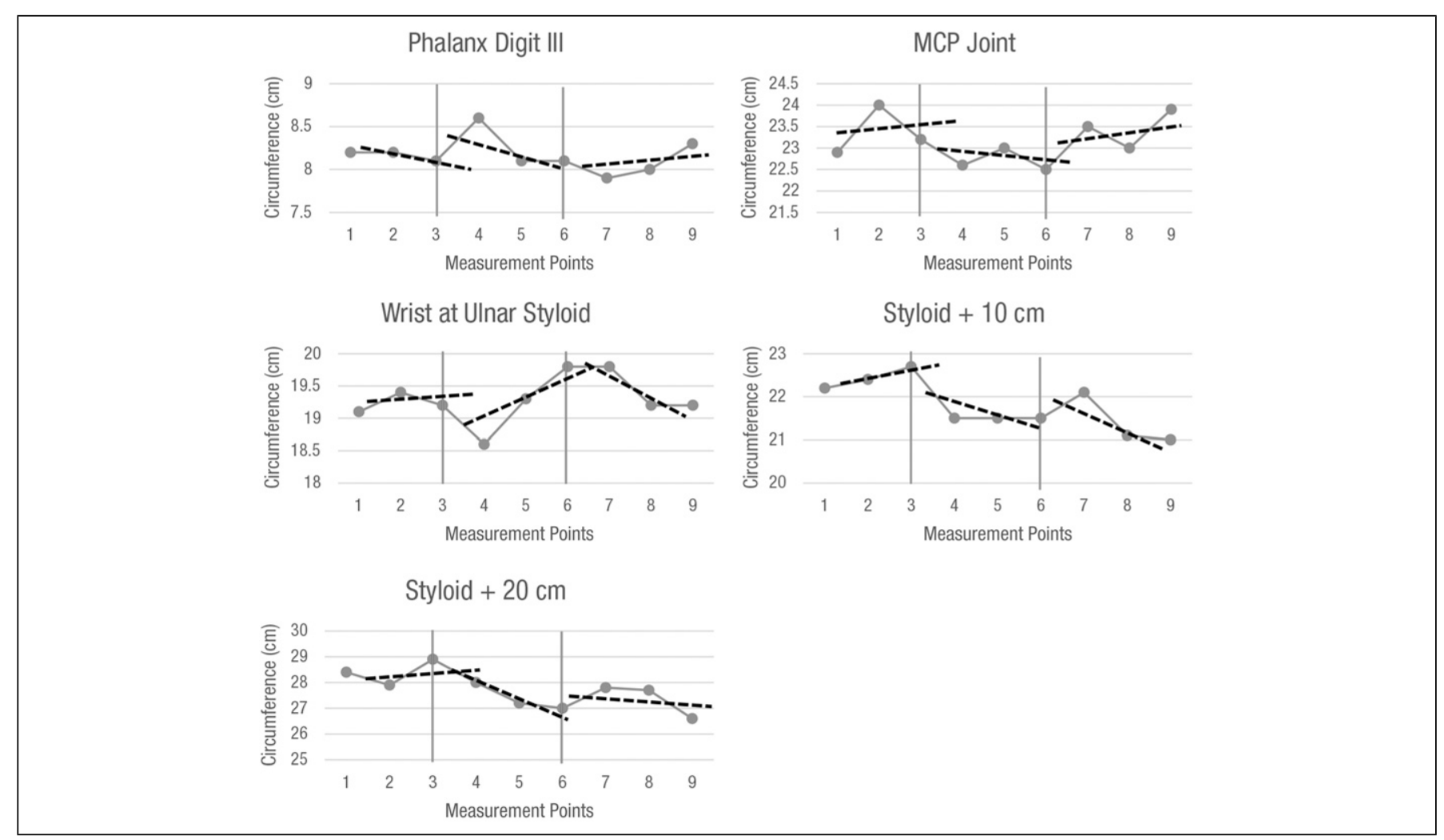

Figure 3. Participant 4.

Note. MCP = metacarpophalangeal.

time consuming when one has a busy clinical workload, but this study suggests that bandaging of the hand alone may be less effective than bandaging of the entire upper limb. Future studies should aim to clearly establish which is more effective in the short and long term to allow a clear demonstration of the health and economic benefits of the more effective method. This is an important area of stroke care, and it is essential that research evaluation of bestpractice approaches continues with the aim of developing an evidence base for poststroke edema management.

\section{Implications for Occupational Therapy Practice}

Implications of this study for occupational therapy practice are as follows:

- Compression bandaging of the entire upper limb may be an effective intervention option for reducing poststroke edema.

- Individual client differences support the need for careful monitoring of edema during and after compression bandaging.

\section{Conclusion}

Bandaging from the fingers to the axilla facilitated a decreasing trend in poststroke edema in the hand and forearm during treatment. The fluctuating nature of edema and individual responses demonstrated by the participants highlight the complexity of edema management. This study is an important first step in the exploration of best practice for the use of compression bandages in poststroke edema, and further research with a larger sample size and statistical evaluation should be undertaken to establish a more comprehensive knowledge and evidence base for poststroke edema management. Future research also needs to examine the impact of full-arm bandaging on engagement in rehabilitation activities to promote sensory and motor return.

\section{Acknowledgments}

This work was partially supported by the National Stroke Foundation (Honours Research Grant 2012). The authors thank the participants for their participation in this study and also acknowledge the Princess Alexandra Hospital, specifically the occupational therapists involved in data collection. The authors declare that there is no conflict of interest.

\section{References}

Badger, C. M. A., Peacock, J. L., \& Mortimer, P. S. (2000). A randomized, controlled, parallel-group clinical trial comparing multilayer bandaging followed by hosiery versus hosiery alone in the treatment of patients with lymphedema of the limb. Cancer, 88, 2832-2837. http://dx.doi.org/10.1002/ 
1097-0142(20000615)88:12<2832::AID-CNCR24>3.0. $\mathrm{CO} ; 2-\mathrm{U}$

Benbow, M. (2009). Managing secondary lymphedema. Journal of Community Nursing, 23(10), 23-30.

Boomkamp-Koppen, H. G., Visser-Meily, J. M., Post, M. W., \& Prevo, A. J. (2005). Poststroke hand swelling and oedema: Prevalence and relationship with impairment and disability. Clinical Rehabilitation, 19, 552-559. http://dx. doi.org/10.1191/0269215505cr846oa

Cheville, A. L., McGarvey, C. L., Petrek, J. A., Russo, S. A., Taylor, M. E., \& Thiadens, S. R. (2003). Lymphedema management. Seminars in Radiation Oncology, 13, 290-301. http://dx.doi.org/10.1016/S1053-4296(03)00035-3

Cohen, S. R., Payne, D. K., \& Tunkel, R. S. (2001). Lymphedema: Strategies for management. Cancer, 92(Suppl.), 980-987. http://dx.doi.org/10.1002/1097-0142(20010815) 92:4+<980::AID-CNCR1410>3.0.CO;2-E

Foley, N., Mehta, S., Jutai, J., Staines, E., \& Teasell, R. (2013). Upper extremity interventions. Retrieved from http:/www.ebrsr.com/sites/default/files/Module-10-upperextremity_FINAL_16ed.pdf

Gebruers, N., Truijen, S., Engelborghs, S., \& De Deyn, P. P. (2011). Incidence of upper limb oedema in patients with acute hemiparetic stroke. Disability and Rehabilitation, 33, 1791-1796. http://dx.doi.org/10.3109/ 09638288.2010 .548895

Geurts, A. C., Visschers, B. A., van Limbeek, J., \& Ribbers, G. M. (2000). Systematic review of aetiology and treatment of post-stroke hand oedema and shoulder-hand syndrome. Scandinavian Journal of Rehabilitation Medicine, 32, 4-10. http://dx.doi.org/10.1080/003655000750045668

Gracies, J. M., Marosszeky, J. E., Renton, R., Sandanam, J., Gandevia, S. C., \& Burke, D. (2000). Short-term effects of dynamic Lycra splints on upper limb in hemiplegic patients. Archives of Physical Medicine and Rehabilitation, 81, 1547-1555. http://dx.doi.org/10.1053/apmr.2000.16346

Gustafsson, L., Patterson, E., Marshall, K., Bennett, S., \& Bower, K. (2016). Efficacy of compression garments in maintaining edema reductions gained after application of compression bandaging to the stroke-affected upper limb. American Journal of Occupational Therapy, 70, 7002290030. http://dx.doi.org/10.5014/ajot.2016.019125
Gustafsson, L., Walter, A., Bower, K., Slaughter, A., \& Hoyle, M. (2014). Single-case design evaluation of compression therapy for edema of the stroke-affected hand. American Journal of Occupational Therapy, 68, 203-211. http://dx. doi.org/10.5014/ajot.2014.009423

Gustafsson, L., \& Yates, K. (2009). Are we applying interventions with research evidence when targeting secondary complications of the stroke-affected upper limb. Australian Occupational Therapy Journal, 56, 428-435. http:// dx.doi.org/10.1111/j.1440-1630.2008.00757.x

Karges, J. R., Mark, B. E., Stikeleather, S. J., \& Worrell, T. W. (2003). Concurrent validity of upper-extremity volume estimates: Comparison of calculated volume derived from girth measurements and water displacement volume. Physical Therapy, 83, 134-145.

Kuppens, S. P., Pijlman, H. C., Hitters, M. W., \& van Heugten, C. M. (2014). Prevention and treatment of hand oedema after stroke. Disability and Rehabilitation, 36, 900-906. http://dx.doi.org/10.3109/09638288.2013.824031

Lymphoedema Framework. (2006). International consensus: Best practice for the management of lymphoedema. Retrieved from http://www.woundsinternational.com/pdf/content_ 175.pdf

National Stroke Foundation. (2010). Clinical guidelines for stroke management 2010. Retrieved from https://strokefoundation. com.au/ /media/strokewebsite/resources/treatment/clinical_ guidelines_stroke_managment_2010_interactive.ashx?la=en

Portney, L. G., \& Watkins, M. P. (2009). Foundations of clinical research: Applications to practice (3rd ed.). Upper Saddle River, NJ: Prentice Hall.

Post, M. W., Visser-Meily, J. M., Boomkamp-Koppen, H. G., \& Prevo, A. J. (2003). Assessment of oedema in stroke patients: Comparison of visual inspection by therapists and volumetric assessment. Disability and Rehabilitation, 25, 1265-1270. http://dx.doi.org/10.1080/09638280310001603992

te Slaa, A., Mulder, P., Dolmans, D., Castenmiller, P., Ho, G., \& van der Laan, L. (2011). Reliability and reproducibility of a clinical application of a simple technique for repeated circumferential leg measurements. Phlebology, 26, 14-19. http://dx.doi.org/10.1258/phleb.2009.009073

Wigg, J., \& Jones, K. (2006). Recognising and managing lymphedema. Practice Nurse, 32, 22-30. 
Reproduced with permission of the copyright owner. Further reproduction prohibited without permission. 